\title{
Thymectomy with angioplasty through a thoracoscopic subxiphoid approach with double elevation of the sternum in Masaoka stage III thymoma
}

\author{
Xing Wang, MD, ${ }^{\mathrm{a}, \mathrm{b}}$ Beatrice Aramini, MD, ${ }^{\mathrm{c}}$ Honglei Xu, MD, and Jiang Fan, MD, ${ }^{\mathrm{a}, \mathrm{b}}$ Shanghai, China, and \\ Modena, Italy
}

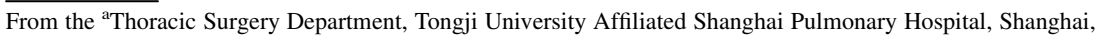
China; 'Thoracic Surgery Department, Shanghai First People's Hospital, Shanghai Jiao Tong University, Shanghai, China; and ${ }^{\mathrm{c} D i v i s i o n}$ of Thoracic Surgery, Department of Medical and Surgical Sciences for Children and Adults, University of Modena and Reggio Emilia, Modena, Italy.

This work was funded by the National Natural Science Foundation of China (81870008).

Disclosures: The authors reported no conflicts of interest.

The Journal policy requires editors and reviewers to disclose conflicts of interest and to decline handling or reviewing manuscripts for which they may have a conflict of interest. The editors and reviewers of this article have no conflicts of interest.

Drs Wang and Aramini contributed equally to this study and should be considered co-first authors.

Read at the 101st Annual Meeting of The American Association for Thoracic Surgery: A Virtual Learning Experience, April 30-May 2, 2021.

Received for publication March 29, 2021; accepted for publication April 7, 2021; available ahead of print April $10,2021$.

Address for reprints: Jiang Fan, MD, 507 Zhengmin Rd, Yangpu District, Shanghai 200433, China (E-mail: fan_jiang@tongji.edu.cn).

JTCVS Techniques 2021;8:208-10

2666-2507

Copyright (c) 2021 The Authors. Published by Elsevier Inc. on behalf of The American Association for Thoracic Surgery. This is an open access article under the CC BY-NC-ND license (http://creativecommons.org/licenses/bync-nd/4.0/).

https://doi.org/10.1016/j.xjtc.2021.04.005
}

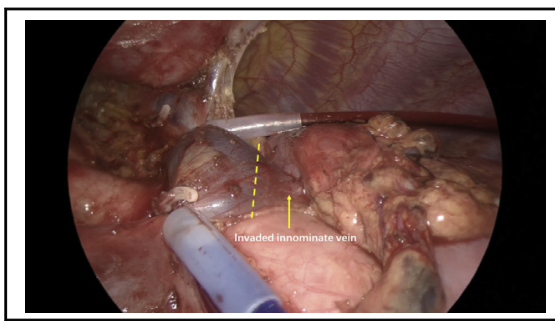

Thymectomy through a subxiphoid approach is feasible in Masaoka stage III thymoma.

CENTRAL MESSAGE

Thymectomy with angioplasty through a subxiphoid approach with double elevation of the sternum might have advantages in Masaoka stage III thymoma and could be recommended as an initial attempt.

See Commentary on page 211.
Subxiphoid thymectomy with double elevation of the sternum is a novel minimally invasive method that might provide a good surgical view and space for operating. ${ }^{1,2}$ Here we report the use of this approach to manage a Masaoka stage III thymoma with invasion to the edge of the innominate vein, which proved safe and feasible.

The Ethics Committee of Shanghai Pulmonary Hospital Affiliated to Tongji University approved the study (L20379; August 27, 2020). The hospital's Institutional Review Board/Ethics Review Board waived the need for informed written consent for publication.

\section{CASE SUMMARY}

This case involves a 66-year-old female patient with an anterior mediastinal mass with a diameter of $5 \mathrm{~cm}$, detected incidentally during physical examination and diagnosed as thymoma. The subxiphoid thymectomy with double elevation of the sternum was performed as described by Zieliński and colleagues. ${ }^{2}$ The patient was placed in a supine position, and a 4-cm vertical incision was made approximately $1 \mathrm{~cm}$ caudal to the xiphoid process to serve as the main operating port. An additional 2-cm skin incision was made between the fourth intercostal anterior axillary lines on the right side for the thoracoscope. A 1-cm incision was made at the level of the jugular process to place hooks. In this way, the sternum could be elevated and the anterior mediastinal space enlarged (Figure 1). (Sometimes the thoracoscope can be placed into the xiphoid port for a better view of the left side of the mediastinum, as for visualizing the left phrenic nerve.)

The surgeon opened the right mediastinal pleura, dissected the connective tissue around the subxiphoid process, and continued to open the right mediastinal pleura upward. The surgeon then detached the thymus and proceeded along the innominate vein by closing the thymic veins with vascular clips until the left internal thoracic vein was visualized. The superficial adipose tissues were gradually detached from the area to expose the distal side of the left 


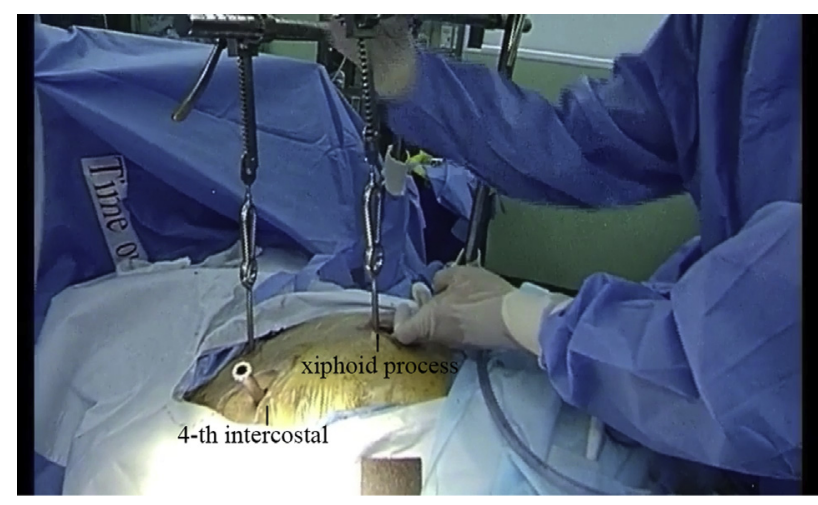

FIGURE 1. Incision strategy.

brachiocephalic vein safely. The superior pole of the thymus and the cervical adipose tissues were dissected from the right brachiocephalic vein on the right side.

To guarantee negative margins, the fat and thymus tissue on the inside of the bilateral phrenic nerve were removed completely, as was the invaded pericardial tissue, in agreement with the International Thymic Malignancy Interest Group criteria. ${ }^{3}$ During surgery, we found that the tumor had invaded the edge of the innominate vein but was not surrounding it. We then attempted to block and control the right and left innominate veins before excising the invaded part (Figure 2). After the en bloc resection of the thymus, a specimen bag was used to remove the resected tissue. Heparin solution was used to flush the inner wall of the innominate vein before continuous suturing (Figure 3), after which the blocker was removed. The surgery lasted $108 \mathrm{mi}-$ nutes, and the patient recovered well and was discharged on day 3 after surgery. The pathology report showed a B2 thymoma with the margin on the innominate vein negative.

\section{DISCUSSION}

In the current clinical guidelines and recommendations, Masaoka stage III thymoma is a contraindication for minimally invasive surgery. ${ }^{4}$ To meet the safety and radical

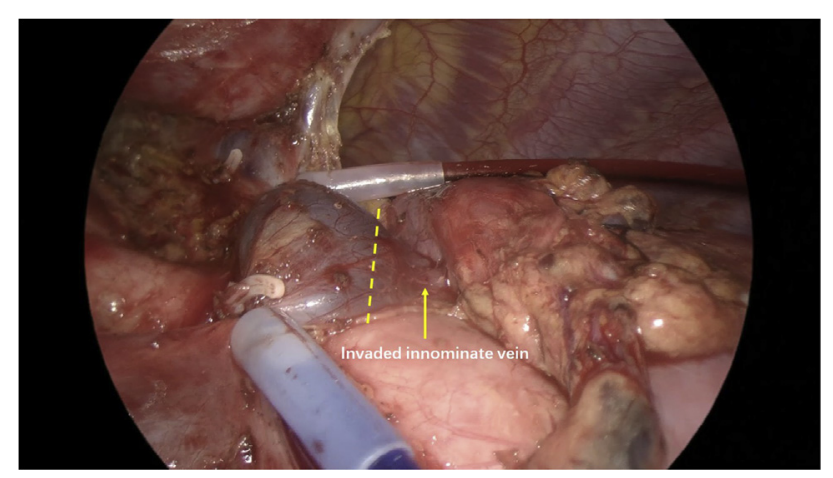

FIGURE 2. The left innominate vein was invaded by tumor (yellow arrow); the dotted line marks the cutting line of the vein.

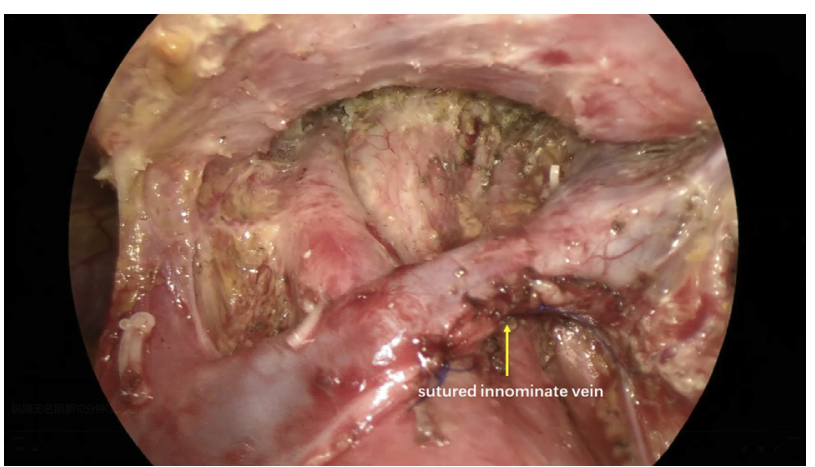

FIGURE 3. Sutured innominate vein and skeletal superior vena cava, brachiocephalic artery, and bronchus.

surgical requirements, open surgery is recommended, and the application of subxiphoid extended thymectomy with double elevation of the sternum in such patients is controversial.

After training on more than 100 cases of subxiphoid extended thymectomy with double elevation, our group believes that this operation pattern is safe and feasible and might be used in Masaoka stage III thymomas. It has several advantages. First, it can fully expose the lateral wall of the innominate vein and vena cava using energy devices for controlling distal and proximal ends; second, double retractors can provide a sufficient visual field and operating space to suture the blood vessels; and third, the depth of the thorax can be increased to facilitate the placement of devices such as staplers and provide space for the resection of bilateral lung tissue that has been invaded. We did not use the stapler across a small portion of vein because it would be close to the tumor edge, although in some cases we did use a stapler.

The good bleeding control afforded by this procedure may give surgeons more confidence in handling Masaoka III stage tumors. The patient is usually placed in a horizontal position with double elevation of the subxiphoid process, and the posterior sternal space is dissected first. As a result,

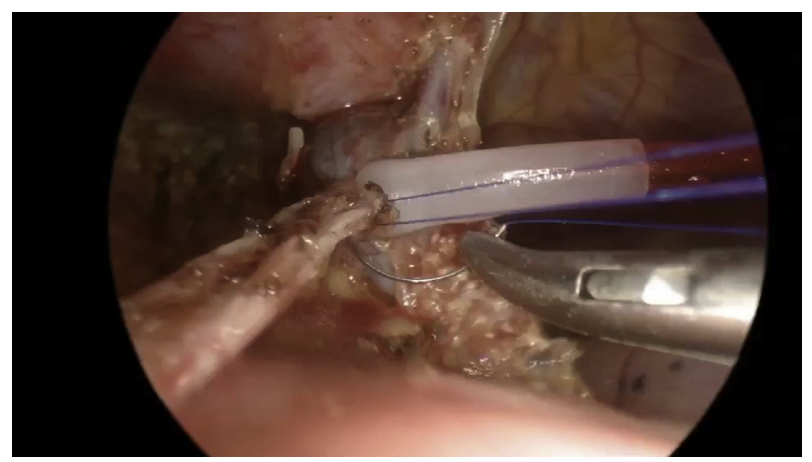

VIDEO 1. The step-by-step surgical procedure for a thymectomy with angioplasty through the subxiphoid approach with double elevation of the sternum in Masaoka stage III thymoma. Video available at: https://www. jtcvs.org/article/S2666-2507(21)00296-0/fulltext. 
surgeons can transfer to median sternotomy quickly and cope with the bleeding sites. In contrast, when using the intercostal approach with the patient in a lateral position, the surgeon might need more time to alter their position, delaying bleeding control. ${ }^{5}$ At present, we do not recommend the subxiphoid approach for routine thymoma treatment in all Masaoka stage III patients and recommend it only in certain situations, such as when imaging suggests potential invasion of the innominate vein, superior vena cava, pericardium, and lungs; otherwise, median sternotomy should be considered first (Video 1).

This case indicates that thymectomy by the subxiphoid approach with double elevation of the sternum might have advantages in Masaoka stage III thymomas, and we recommend this approach for the initial attempt for potential Masaoka stage III thymoma. However, immediate conversion to open surgery should be performed for incomplete resection or intraoperative accidents, and more evidence should be accumulated.

\section{References}

1. Aramini B, Song N, Banchelli F, Jiang G, Gonzalez-Rivas D, Fan J. Subxiphoid thymectomy with a double sternum retractor: a pilot study. Gland Surg. 2019;8: 657-62.

2. Zieliński M, Rybak M, Solarczyk-Bombik K, Wilkojc M, Czajkowski W, Kosinski S, et al. Subxiphoid uniportal VATS thymectomy. J Vis Surg. 2017;3: 171.

3. Marx A, Ströbel P, Badve SS, Chalabreysse L, Chan JKC, Chen G, et al. ITMIG consensus statement on the use of the WHO histological classification of thymoma and thymic carcinoma: refined definitions, histological criteria, and reporting. $J$ Thorac Oncol. 2014;9:596-611.

4. Jiang JH, Gao J, Zhang Y, Wang H, Tan LJ, Ding JY. Modified subxiphoid thoracoscopic thymectomy for locally invasive thymoma. Ann Thorac Surg. November 26, 2020 [Epub ahead of print].

5. Hirai K, Ibi T, Bessho R, Koizumi K, Shimizu K. Video-assisted thoracoscopic thymectomy (VAT-T) with lateral thoracotomy for stage II and III thymoma. Ann Thorac Cardiovasc Surg. 2013;19:79-82. 